\title{
PENALIZED SPLINE: A GENERAL ROBUST TRAJECTORY MODEL FOR ZIYUAN-3 SATELLITE
}

\author{
H. Pan, Z. Zou \\ School of Geosciences and Info-Physics, Central South University, Changsha 410083, China - \\ (hongbopan,zrzou) @csu.edu.cn
}

Commission I, WG I/4

KEY WORDS: Penalized Spline, Trajectory Model, ZiYuan-3 Satellite, Band-to-band Registration, Multispectral Images

\begin{abstract}
:
Owing to the dynamic imaging system, the trajectory model plays a very important role in the geometric processing of high resolution satellite imagery. However, establishing a trajectory model is difficult when only discrete and noisy data are available. In this manuscript, we proposed a general robust trajectory model, the penalized spline model, which could fit trajectory data well and smooth noise. The penalized parameter $\lambda$ controlling the smooth and fitting accuracy could be estimated by generalized crossvalidation. Five other trajectory models, including third-order polynomials, Chebyshev polynomials, linear interpolation, Lagrange interpolation and cubic spline, are compared with the penalized spline model. Both the sophisticated ephemeris and on-board ephemeris are used to compare the orbit models. The penalized spline model could smooth part of noise, and accuracy would decrease as the orbit length increases. The band-to-band misregistration of ZiYuan-3 Dengfeng and Faizabad multispectral images is used to evaluate the proposed method. With the Dengfeng dataset, the third-order polynomials and Chebyshev approximation could not model the oscillation, and introduce misregistration of 0.57 pixels misregistration in across-track direction and 0.33 pixels in along-track direction. With the Faizabad dataset, the linear interpolation, Lagrange interpolation and cubic spline model suffer from noise, introducing larger misregistration than the approximation models. Experimental results suggest the penalized spline model could model the oscillation and smooth noise.
\end{abstract}

\section{INTRODUCTION}

Different from frame cameras, dynamic imaging system utilizes the relative movement between cameras and targets to capture two dimensional (2D) images. The trajectory model of linear pushbroom cameras, like ZY3, describes satellite movements by attitude and orbit models. The movement is one of the critical issues in modelling the imaging process. The trajectory models of linear pushbroom camera were studied since SPOT-1. However, no consensus has been reached as to handling the attitude data and ephemeris data on board. Some use the attitude data and ephemeris data as the initial value of the simplified models, which usually assume the trajectory model is stable and could be modelled with no more than $3^{\text {rd }}$ polynomials or twobody motion model. This hypothesis is sensible when the accuracy of attitude and orbit data are limited. Some others assume that the attitude data and ephemeris data are with sufficient accuracy, so only compensation models are required to compensate the errors of trajectory models. Since the precise orbit determination and star trackers are used for high resolution satellite, the compensation methods can achieve remarkable accuracy with fewer ground control points (GCPs).

To obtain m-level resolution, the sampling frequency of scan line should be over $1000 \mathrm{~Hz}$. However, the frequency of attitude and orbit data is much lower than the imaging system. To get the position and attitude of each scan line, trajectory models are used. The trajectory models could be classified into two categories: the approximation model and the interpolation model. The ephemeris and attitude records are used to estimate the coefficients of approximation models, such as polynomial models. For interpolation models, records are used as true values, and different interpolation strategies mean different trajectory models. However, there are confliction between these trajectory models. Taking ALOS for example, second order polynomial model is different from linear interpolation model especially when there is oscillation(Schwind et al., 2009). In this paper, we propose the penalized spline model, a new generic robust trajectory model, which could model the oscillation and reduce noise of trajectory data.

Bundle adjustment is one of the most powerful methods to evaluate the trajectory models (Jeong and Bethel, 2010). However, its accuracy is limited by the accuracy of GCPs, especially the points picking accuracy which is about 0.3 pixels. Besides, this method requires a large number of check points (CKPs), which is costly. With high precise orbit determination (POD), the orbit accuracy after post processing could achieve cm-level(Luthcke et al., 2003), which could be used as true value for spaceborne photogrammetric applications. Given that its accuracy is limited by the accuracy of attitude, the band-toband registration (BBR) is used to compare the attitude models.

\section{TRAJECTORY MODELS}

\subsection{Mathematical Problem}

Building the function model with the discrete trajectory data is the mathematical problem. And an interpolation model will be used when the value of trajectory model is exactly equal to the discrete attitude and orbit data. Otherwise, approximation model will be chosen.

There are two kinds of trajectory data for ZY3: on-board and sophisticated. The sophisticated trajectory data is smooth and accurate after post processing. However, it is not always available, especially in emergency. The on-board attitude data is determined by extended Kalman filter (EKF) with star-tracker and gyro data, and the orbit data is determined by dualfrequency GPS receiver. Generally, the on-board trajectory data is studied, which is noisy and discrete. The mathematical model of trajectory data is: 


$$
a_{i}=a\left(t_{i}\right)+\varepsilon
$$

with

$a_{i} \quad$ : discrete trajectory data

$a\left(t_{i}\right):$ trajectory model

$\varepsilon \quad$ : noise

The problem turns to be estimating $a\left(t_{i}\right)$ with discrete trajectory data $a_{i}$.

\subsection{Penalized B-Spline}

Because of the stable numerical properties, B-spline is usually used as the base function for univariate regression. Any spline function of k-degree can be expressed as a linear combination of B-splines of k-degree. With the given set of strictly increasing knots (where distinct B-spline segments join together) $\tau_{l}, l=0, \cdots, g+2 k+1$, the spline has a unique expression:

$$
f(t)=\sum_{j=0}^{g+k} c_{j} B_{j, k+1}(t)
$$

where $c_{j}$ is the B-spline coefficients of $f(t)$, and $B_{j, k+1}(t)$ is the $j$-th B-spline basis function of degree $k$, whose recursion definition is (de Boor, 2001; Dierckx, 1995)

$$
\begin{aligned}
& B_{j, k+1}(t)=\frac{t-\tau_{j}}{\tau_{j+k}-\tau_{j}} B_{j, k}(t)+\frac{\tau_{j+k}-t}{\tau_{j+k+1}-\tau_{j+1}} B_{j+1, k}(t), \quad k>0 \\
& B_{j, 0}(t)=\left\{\begin{array}{cc}
1, & t \in\left[\tau_{j}, \tau_{j+1}\right), \\
0, & \text { otherwise. }
\end{array} \quad, k=0\right.
\end{aligned}
$$

Usually, the coincident boundary knots are chosen to construct knots $\tau_{1}$ as follows:

$$
\tau_{l}=\left\{\begin{array}{cc}
t_{0} & 0 \leq l \leq k \\
\tau_{l} & k<l \leq g+k \\
t_{N} & g+k<l
\end{array}\right.
$$

And the knots should satisfy the Schoenberg-Whitney condition(Dierckx, 1995). Then the coefficients $c_{j}$ with the fixed knots could be calculated by the weighted least square algorithm.

$$
\min _{c}\|w \mathbf{B} c-w \mathbf{a}\|_{2}^{2}
$$

where
$\mathbf{B}=\left[\begin{array}{ccc}w_{0} B_{0,4}\left(t_{0}\right) & \cdots & w_{0} B_{g+k, 4}\left(t_{0}\right) \\ \vdots & & \vdots \\ w_{N} B_{0,4}\left(t_{N}\right) & \cdots & w_{N} B_{g+k, 4}\left(t_{N}\right)\end{array}\right] \quad, \quad \mathbf{a}=\left[\begin{array}{c}w_{0} a_{0} \\ \vdots \\ w_{N} a_{N}\end{array}\right] \quad$ and
$w_{i}, i=0, \cdots, N$ is the weight of attitude $a_{i}$. In this article, third- degree B-splines is used for attitude approximation, in which $k=3$.

The statement above indicate the number and position of these knots greatly impact on the results, to be detailed, too few knots lead to the under fitting of the data while too many knots lead to overfitting. However, solving B-splines with variable knots becomes rather complex because it is a non-linearly leastsquares problem(de Boor, 2001; Dierckx, 1995).

Penalized Spline is a good solution to this problem, which adds a penalty to the residual sum of squares.

$$
S=\sum_{i=0}^{N} w_{i}^{2}\left\{a_{i}-w_{j} f\left(t_{j}\right)\right\}^{2}+\lambda P(t)
$$

where $\lambda$ is the penalty factor, the first term measuring the closeness of fitting, and $P(t)$ is the penalty function, a measure of smoothness. Finite differences of coefficients of adjacent Bsplines is a flexible discrete penalty(Eilers and Marx, 1996). This penalty defines the second-degree derivative matrix $\mathbf{D}$ as

$$
\mathbf{D}=\left[\begin{array}{cccccc}
1 & -2 & 1 & 0 & \cdots & 0 \\
0 & 1 & -2 & 1 & \cdots & 0 \\
\vdots & \vdots & \ddots & \ddots & \ddots & \vdots \\
0 & \cdots & 1 & -2 & 1 & 0 \\
0 & \cdots & 0 & 1 & -2 & 1
\end{array}\right]
$$

The corresponding penalty matrix is then

$$
\mathbf{P}=\mathbf{D}^{T} \mathbf{D}
$$

The roughness of penalized splines is controlled by the penalty parameter $\lambda$, and the number of knots is not a crucial parameter. $\lambda$ plays a trade-off roles between smoothing and fitting accuracy. If $\lambda=0$, the approximation becomes a cubic spline interpolation, and the errors are rather small. If $\lambda=\infty$, the approximation becomes the weighted least-squares polynomial of degree k. The solution of Formula (5) is similar to ridge regression. Generalized cross validation(GCV) is used to choose an appropriate $\lambda$ (Golub et al., 1979).

\subsection{Linear Interpolation}

Linear interpolation is based on the hypothesis that the rates between two nodes are constant. The model below, recommended by SPOT(SPOTImage, 2002), is the simplest local interpolation method

$$
f(t)=a_{i} \cdot \frac{t_{i+1}-t}{t_{i+1}-t_{i}}+a_{i+1} \cdot \frac{t-t_{i}}{t_{i+1}-t_{i}}
$$

where $t \in\left[t_{i}, t_{i+1}\right]$.

\subsection{Lagrange Interpolation}

Lagrange interpolation is recommended by Ebner(Ebner et al., 1993). The $n+1$ neighbouring attitudes would be used for $n$ order Lagrange interpolation, which can be written as follows: 


$$
f(t)=\sum_{i=0}^{n} a_{i} \prod_{j=0, j \neq i}^{n} \frac{t-t_{j}}{t_{i}-t_{j}}
$$

when $\mathrm{n}=1$, it equals to the linear interpolation.

\subsection{Polynomials Approximation}

Polynomials are the most common models for attitude (Gugan, 1987; Konecny et al., 1987). Being widely accepted (Li et al., 2011; Radhadevi et al., 1998; Teshima and Iwasaki, 2008), third-order polynomials are used for modelling attitudes, as follows

$$
f(\bar{t})=c_{0}+c_{1} \cdot \bar{t}+c_{2} \cdot \bar{t}^{2}+c_{3} \cdot \bar{t}^{3}
$$

where variable $\bar{t}$ is normalized by start time $t_{s}$ and end time $t_{e}$, defined as:

$$
\bar{t}=\frac{t-t_{s}}{t_{e}-t_{s}} \in[0,1]
$$

and $c_{j}$ is the coefficients of degree $j$. These coefficients could be solved by Least Square method.

\subsection{Chebyshev Approximation}

Chebyshev approximation was recommended by Schwind (Schwind et al., 2012), and used for orbit interpolation of TerraSAR-X(Breit et al., 2010). It is a polynomial of "the best approximation", which minimizes the maximum norm of difference between polynomial of degree $n$ and measurements. And it is orthogonal on $t^{\prime} \in(-1,1)$ with respect to the weight function $w(t)=\left(1-t^{\prime 2}\right)^{-1 / 2}$. The $\mathrm{n}$ degree Chebyshev polynomials are defined as follows:

$$
f\left(t^{\prime}\right)=\sum_{j=0}^{n} c_{j} T_{j}\left(t^{\prime}\right)
$$

where $t^{\prime}$ is the regularized time $t^{\prime}=\frac{2 t-t_{s}-t_{e}}{t_{e}-t_{s}} \in[-1,1], n$ is the degree of Chebyshev approximation, and

$$
T_{j}\left(t^{\prime}\right)=\cos \left[j \arccos t^{\prime}\right] \text {, for each } j \geq 0
$$

Derived from Formula (13), we can get

$$
\begin{aligned}
& T_{0}\left(t^{\prime}\right)=1 \\
& T_{1}\left(t^{\prime}\right)=t^{\prime} \\
& T_{j+1}\left(t^{\prime}\right)=2 t^{\prime} T_{j}\left(t^{\prime}\right)-T_{j-1}\left(t^{\prime}\right), \quad j \geq 1
\end{aligned}
$$

With $N$ nodes $a_{i}, i=0, \cdots, N \quad$, the coefficients $c_{i}, j=0, \cdots, n(n \leq N)$ could be solved by minimizing least square.

\subsection{Hermite and Cubic Splines Interpolation}

Cubic splines interpolation, also called piecewise cubic interpolation, is continuously differentiable on the interval and has continuous second derivate. Comparing with higher degree polynomial interepolation, cubic spline is preferred because of similar results and avoiding instability due to Runge's phenomenon as well. It was adopted by Weser's generic sensor model for pushbroom satellite imagery(Weser et al., 2008).

$$
f_{n}(\bar{t})=c_{0, n}+c_{1, n} \cdot \bar{t}+c_{2, n} \cdot \bar{t}^{2}+c_{3, n} \cdot \bar{t}^{3}
$$

where $n$ is the index of the spline segment, $c_{j, n}$ is the

polynomial coefficients of degree $j$, and $\bar{t}$ has the same definition as that in Formula(11).

Cubic splines could be divided, in terms of different slopes and boundary conditions, into cubic Hermite spline, cubic Bessel spline, cubic Akima spline, complete cubic spline, natural cubic spline, etc.(de Boor, 2001). If the angular rates are available, the angular values and rates of segments can be used to determine the coefficients, which is the Hermite interpolation, or the firstorder osculating interpolation(Tanygin, 2003).

In this paper, natural cubic splines are used for attitude interpolation, where the second derivatives of boundary equals to 0 . If the second derivatives of adjacent splines are continous at the knots, another constraint function could be introduced, and the final equation could be written as:

$$
\begin{aligned}
& c_{0, n}=a_{n}(0) \\
& c_{0, n}+c_{1, n}+c_{2, n}+c_{3, n}=a_{n}(1) \\
& c_{1, n}+2 c_{2, n}+3 c_{3, n}=c_{1, n+1} \\
& 2 c_{2, n}+6 c_{3, n}=2 c_{2, n+1}
\end{aligned}
$$

The problem of coefficients is turned into solving the first derivate, which is a simple tridiagonal system. Hence, natural cubic splines are also a special case of Hermite interpolation. With Formula (16) and boundary condition, the coefficients could be solved.

\section{EXPERIMENTS}

Trajectory models, including linear interpolation, third-order polynomials, Chebyshev approximation, Lagrange interpolation and cubic spline are compared with the proposed penalized spline model.

\subsection{Orbit Models}

Ephemeris data of ZY3 on Aug. 23, 2012 is used in the experiments. Since the approximation models depend on the orbit length, the total 12 scenes are used to evaluate the orbit models. Because the sophisticated ephemeris is discrete, the orbit models are compared to Lagrange interpolation recommended by Toutin (2004). The difference between Lagrange interpolation and other models including penalized spline, Chebyshev approximation and cubic spline model is within $2 \mathrm{~mm}$. We could conclude that they have the same accuracy when sophisticated ephemeris is available/applied. Linear interpolation and third-order polynomials introduce an error of about $0.5 \mathrm{~m}$ in three axes respectively. 
The mean and standard variance (STDEV) of the difference between the orbit models based on on-board data and sophisticated ephemeris are listed in Table 1.

Table 1 Mean value and variance of the differences between the attitude data and sophisticated ephemeris

\begin{tabular}{|c|c|c|c|c|c|c|}
\hline \multirow{2}{*}{$\begin{array}{l}\text { Orbit } \\
\text { Models }\end{array}$} & \multicolumn{2}{|c|}{$X(m)$} & \multicolumn{2}{|c|}{$\mathbf{Y}(\mathbf{m})$} & \multicolumn{2}{|c|}{$\mathrm{Z}(\mathrm{m})$} \\
\hline & Mean & STDEV & Mean & STDEV & Mean & STDEV \\
\hline $\begin{array}{l}\text { Penalized } \\
\text { Spline }\end{array}$ & 0.09 & 0.29 & 0.82 & 0.54 & 3.60 & 0.48 \\
\hline $\begin{array}{l}\text { Third-order } \\
\text { Polynomials }\end{array}$ & 0.14 & 0.49 & 0.79 & 0.65 & 3.55 & 0.59 \\
\hline $\begin{array}{l}\text { Chebyshev } \\
\text { Polynomials }\end{array}$ & 0.04 & 0.26 & 1.03 & 0.55 & 3.51 & 0.39 \\
\hline $\begin{array}{c}\text { Linear } \\
\text { Interpolation }\end{array}$ & 0.40 & 0.49 & 0.35 & 0.89 & 3.16 & 0.88 \\
\hline $\begin{array}{c}\text { Lagrange } \\
\text { Interpolation }\end{array}$ & 0.10 & 0.51 & 0.83 & 0.93 & 3.60 & 0.93 \\
\hline Cubic Spline & 0.10 & 0.54 & 0.83 & 0.99 & 3.60 & 1.00 \\
\hline
\end{tabular}

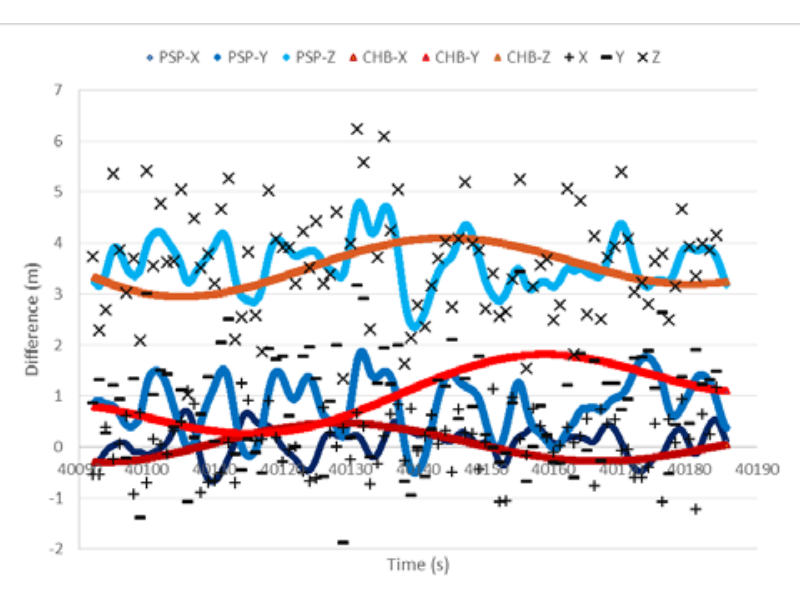

Figure 1. Differences between the attitude data and sophisticated ephemeris.

Chebyshev approximation has the minimum STDEV, whereas the cubic spline has the largest variance. If we look insight into the differences, Chebyshev polynomials have a smoother result than the penalized spline (see Figure. 1) as the former have less parameters. However, it would introduce larger errors if the orbit becomes longer. And for this reason, the STDEV of thirdorder polynomials is larger than Chebyshev polynomials. The interpolation models, including linear interpolation, Lagrange interpolation and cubic spline interpolation have larger variance, since they use the on-board data as the true value. Differences between the on-broad data and sophisticated ephemeris are illustrated as $\mathrm{X}, \mathrm{Y}, \mathrm{Z}$ in Figure 1. There are significant noise, and the result in Table 1 is expectable. The penalized spline model has smaller STDEV than the interpolation models, because the GCV are used to estimate the noise. Owing to inaccurate noise estimation, the penalized parameter $\lambda$ is underestimated.

However, if the orbit length is extended to 30 scenes (about 222 seconds), STDEV of Chebyshev polynomials in the three axes become $0.33 \mathrm{~m}, 1.69 \mathrm{~m}$ and $1.66 \mathrm{~m}$ respectively, whereas STDEV of the penalized spline model are about $0.28 \mathrm{~m}, 0.62 \mathrm{~m}$ and $0.50 \mathrm{~m}$ respectively. In the case of standard scene, the best result is generated by third-order polynomials, whose STDEV in three axes become $0.03 \mathrm{~m}, 0.11 \mathrm{~m}$ and $0.19 \mathrm{~m}$ respectively, which means the orbit within 8 seconds could be fitted by thirdpolynomials with a very high accuracy.

\subsection{Attitude Models}

The Dengfeng dataset and Faizabad dataset are used in the experiment. The Dengfeng dataset was obtained on 02/03/2012 and the Faizabad dataset was obtained on 9/01/2013. The two scenes have abundant textures, which could have high accurate BBR accuracy when phase correlation is used to determine the shift between two bands.

The BBR of ZY3 multispectral images is influenced by interior orientation parameter (IOP) errors, attitude errors and height errors (Pan et al., 2016). Since each scanline has the same attitude errors and each column has the same IOP errors, the average misregistration is used to evaluate the IOP error and attitude errors. The pitch angle and roll angle play a much more important role than yaw angle for narrow field of view push broom cameras.

The oscillation problem, also called "jitter" in some articles, is common for linear pushbroom cameras. Utilizing the BBR of ZY3 multispectral raw data, Tong et al. (2015) proved that there is oscillation problem for ZY3 multispectral data. However, after sensor correction procedure, the misregistration of ZY3 is not severe. It means the oscillation has been detected by the gyros and star-trackers. The frequency of ZY3 attitude is $4 \mathrm{~Hz}$, higher than the Nyquist sampling frequency of the oscillation. Therefore, the misregistration is caused by the attitude model and noise.

Third-order polynomials are used as attitude model. The misregistration of the Dengfeng dataset and Faizabad dataset are illustrated in Figure 2 and 3. As the figures show, there are significant oscillations, and the frequencies of the oscillations are different between the two datasets. Third-order polynomials could not model oscillations, even detected by attitude measurements.

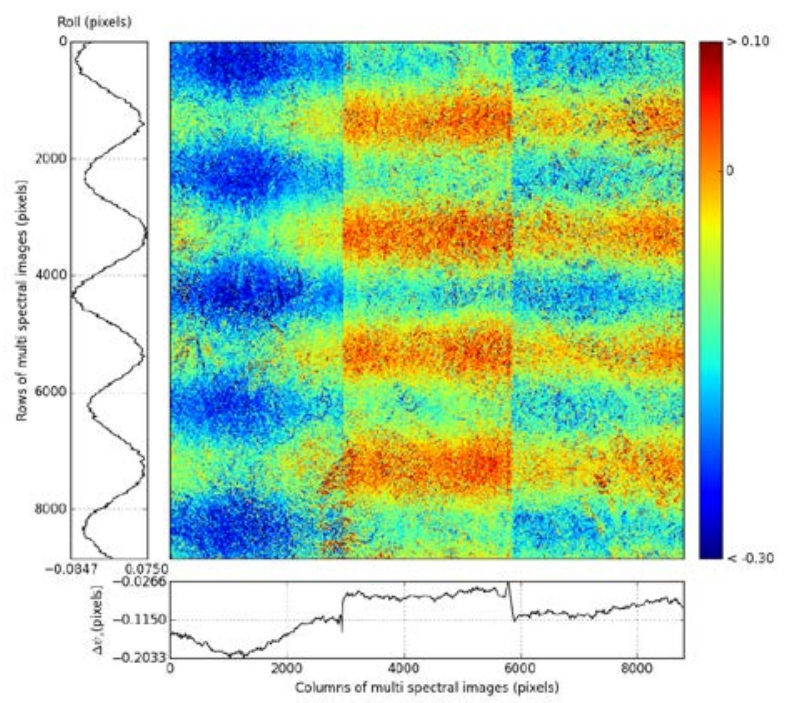

(a) Across-track 


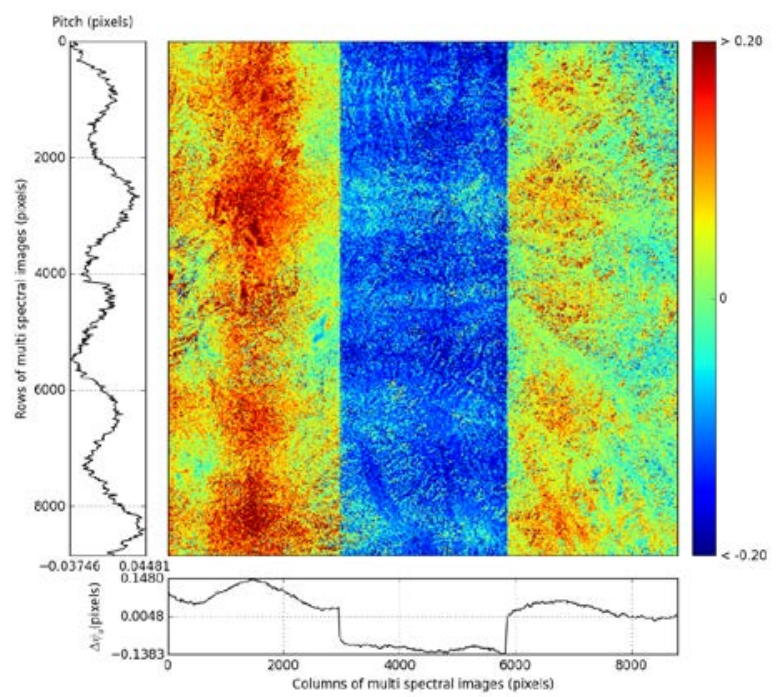

(b) Along-track

Figure 2 The band-to-band misregistration of Faizabad dataset with third-order polynomials.

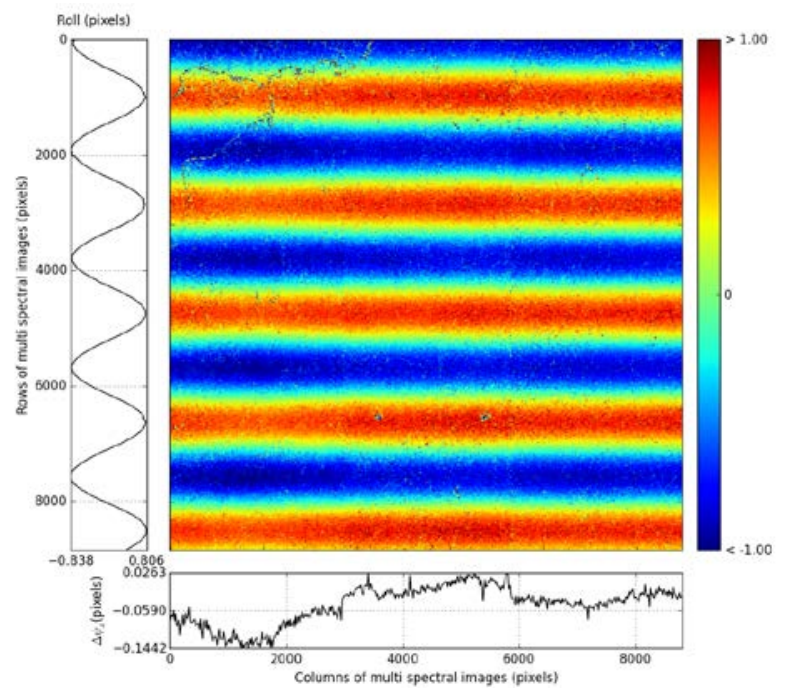

(a) Across-track

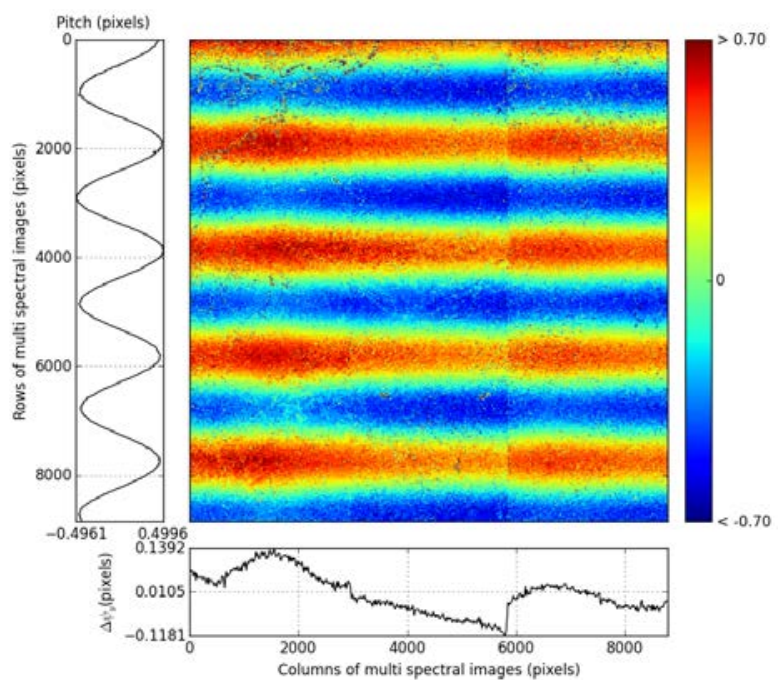

(b) Along-track

Figure 3 Band-to-band misregistration of Dengfeng dataset with third-order polynomials.
Misregistration in the across-track direction is between -0.84 pixels to 0.81 pixels, while the along-track is between -0.50 pixels to 0.50 pixels. However, the oscillation in the Faizabad dataset is much smaller than that of the Dengfeng dataset. And the IOP errors of Faizabad dataset could be identified in both the across-track and along-track direction. The IOP error of the two datasets are different, especially between CCDs. The IOP of ZY3 multispectral changes over time.

Cubic spline, a classic interpolation model, is used as an attitude model in the experiment. Then the misregistration of the Dengfeng dataset and Faizabad dataset are shown in Figure 4 and 5. More strips could be identified in both these two datasets. Compared with that of third-order polynomials, the misregistration of the Faizabad dataset is larger in both the along and across track directions. The noise of attitude data causes misregistration. In the first 2000 rows along the track, the misregistration ranges from -0.15 to 0.12 pixels, much larger than third-order polynomials. On the contrary, the misregistration of cubic spline is much smaller than that of third-order polynomials, meaning the cubic spline model could model the oscillation. And the residual misregistration in both along and across track direction is from -0.12 to 0.12 pixels.

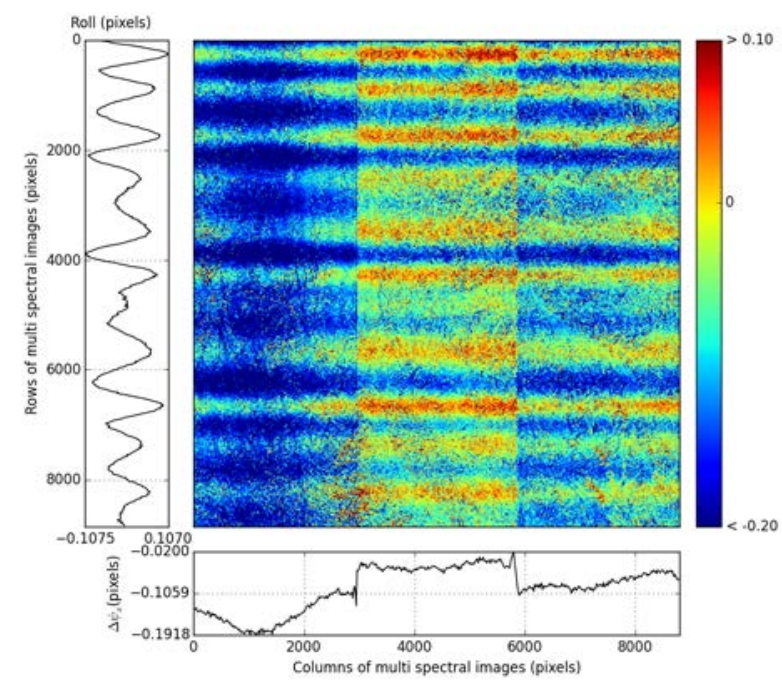

(a) Across-track

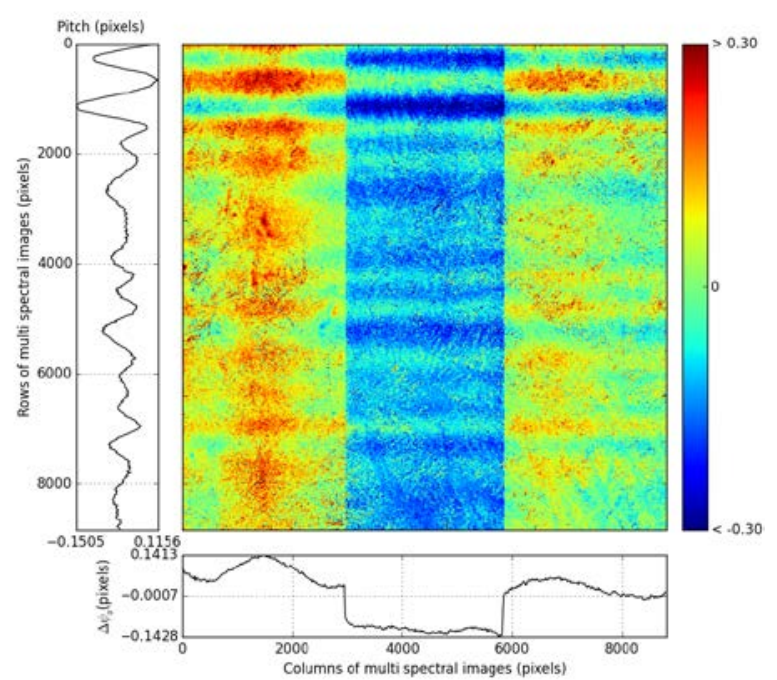

(b) Along-track

Figure 4 Band-to-band misregistration of Faizabad dataset with cubic spline. 


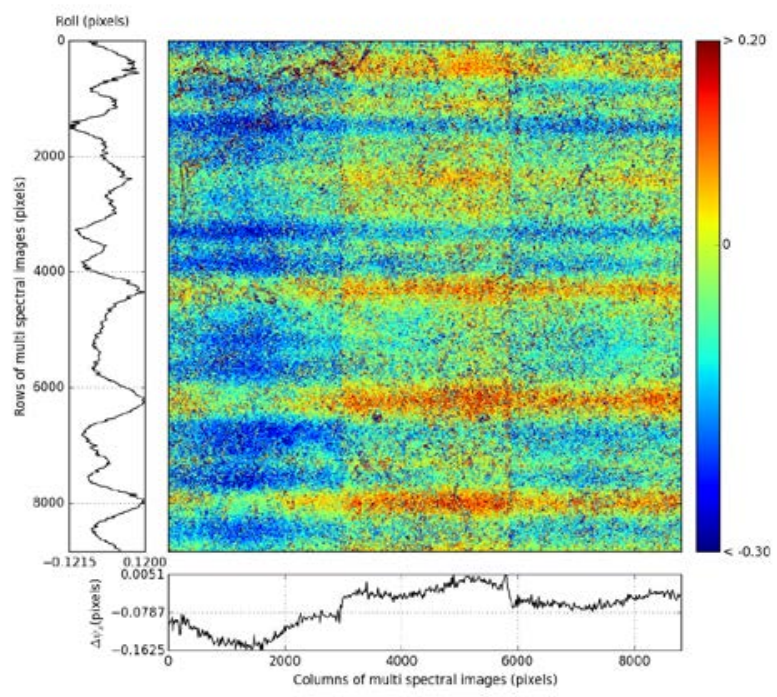

(a) Across-track

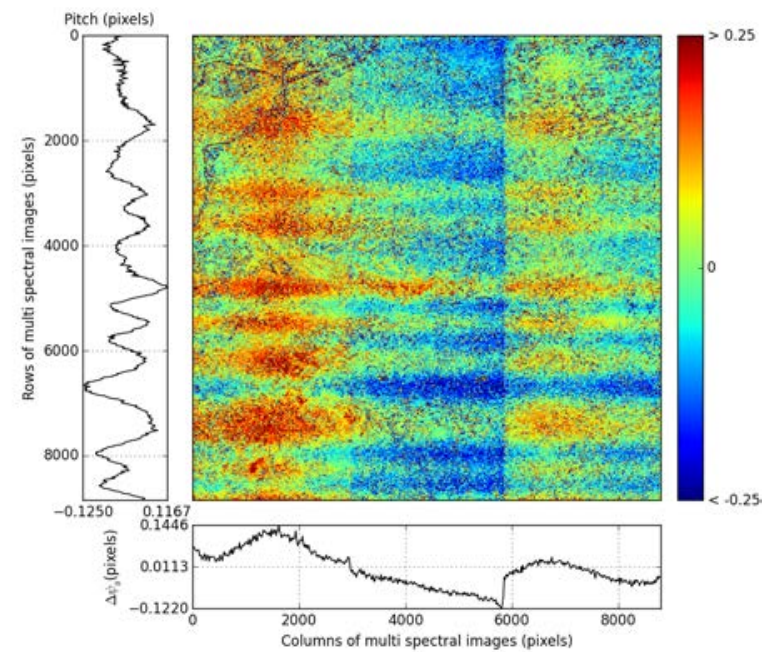

(b) Along-track

Figure 5 The band-to-band misregistration of Dengfeng dataset with cubic spline

When the penalized spline is used to model attitude, the misregistration of Faizabad dataset is reduced to 0.05 pixels, smaller than that of both the third-order polynomials and cubic spline. But its result of the Dengfeng dataset is close to that of the cubic spline model. Since the oscillation in the Dengfeng dataset shows a very high signal to noise ratio, it is difficult to estimate the noise when signal dominate the attitude data.

In the Faizabad dataset, interpolation models suffer from noises, as illustrated in Figure 7. The root mean square errors (RMSE) of misrigstration caused by attitude models are calculated and shown in Table 2. The performance of linear interpolation is better than the Lagrange interpolation and cubic spline model. The third-order polynomials have the same result as the Chebyshev approximation. Due to larger oscillations in the roll angle, apprximation models have similar RMSEs as interpolation models, but with a better performance in the along-track direction. The best result is derived from the penalized spline model, which could model the attitude oscillation and reduce noises both along and across the track.

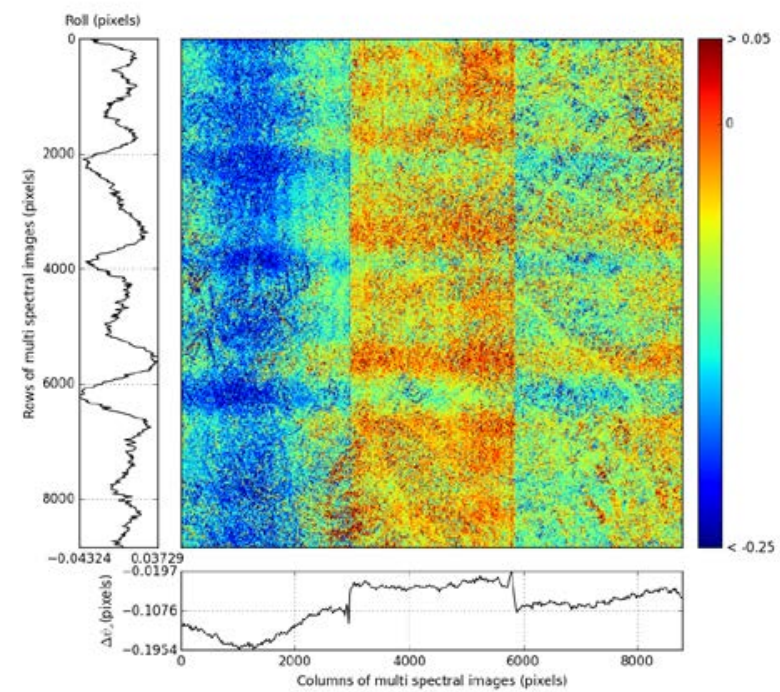

(a) Across-track

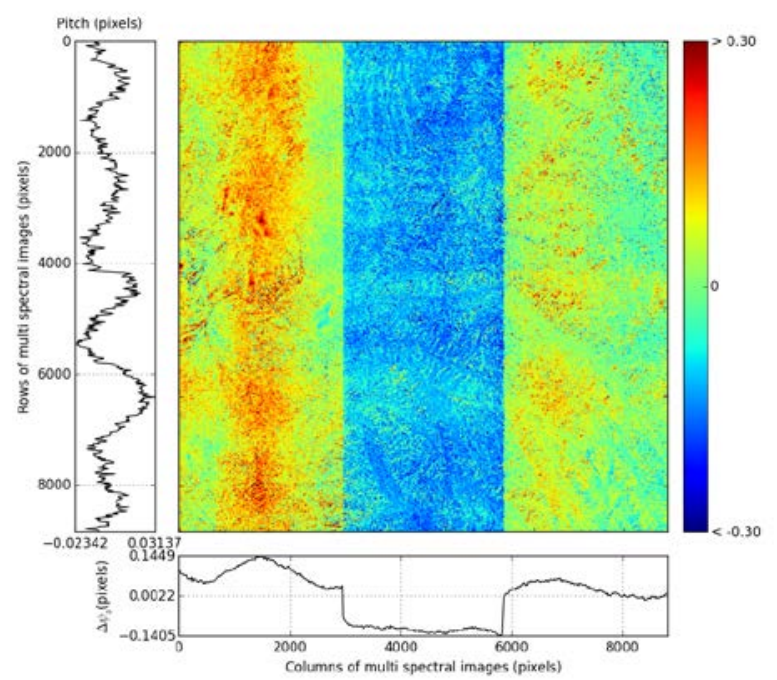

(b) Along-track

Figure 6 Band-to-band misregistration of Faizabad dataset with penalized spline.

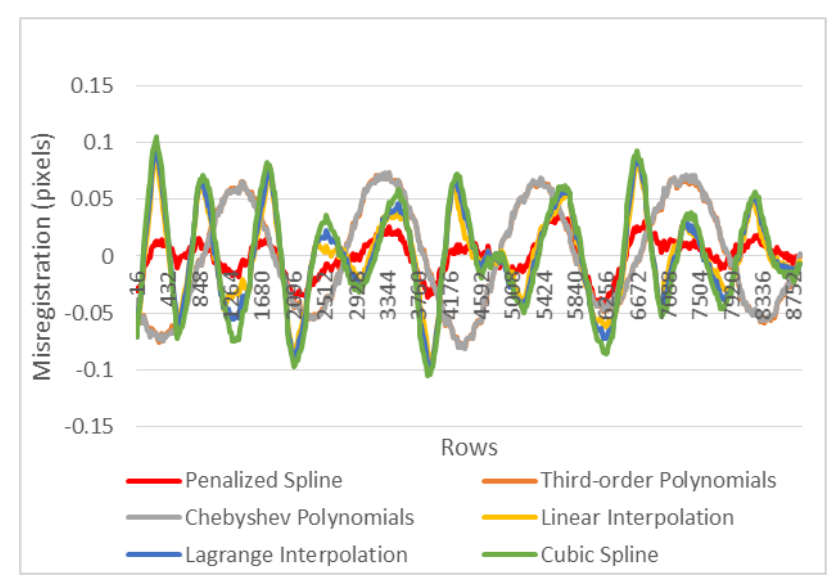

(a) Across-track 


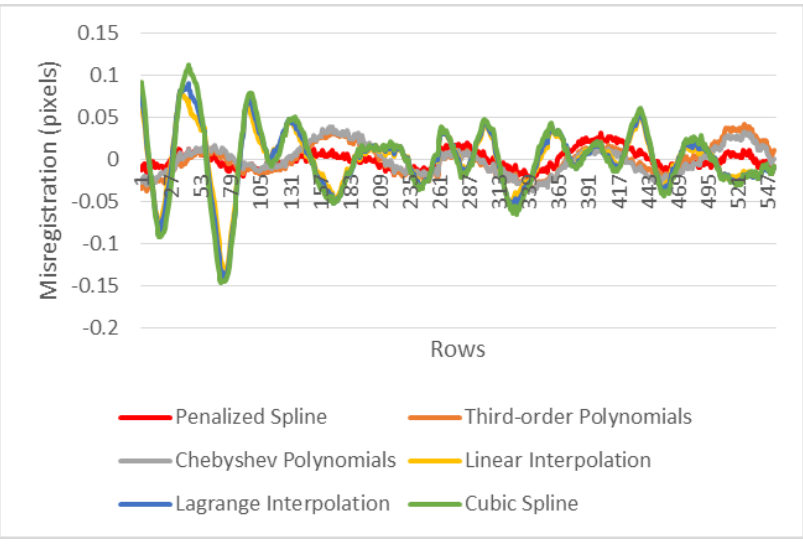

(b) Along-track

Figure 7 Band-to-band misregistration of Faizabad dataset caused by the attitude errors.

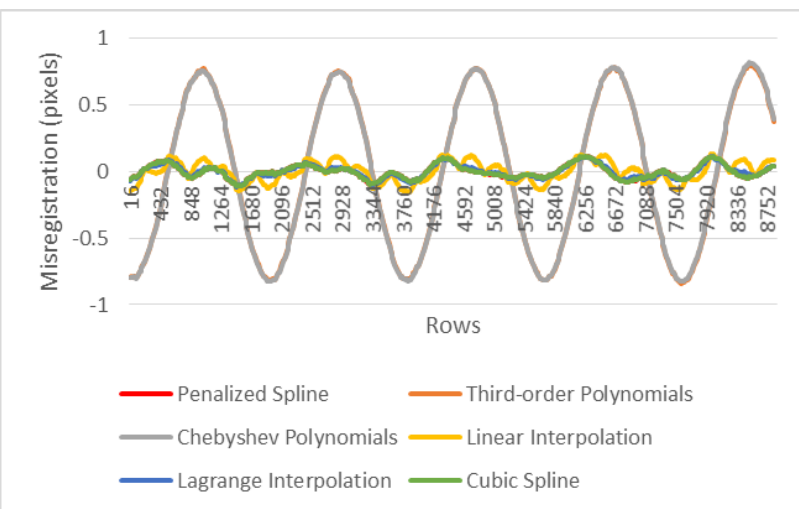

(a) Across-track

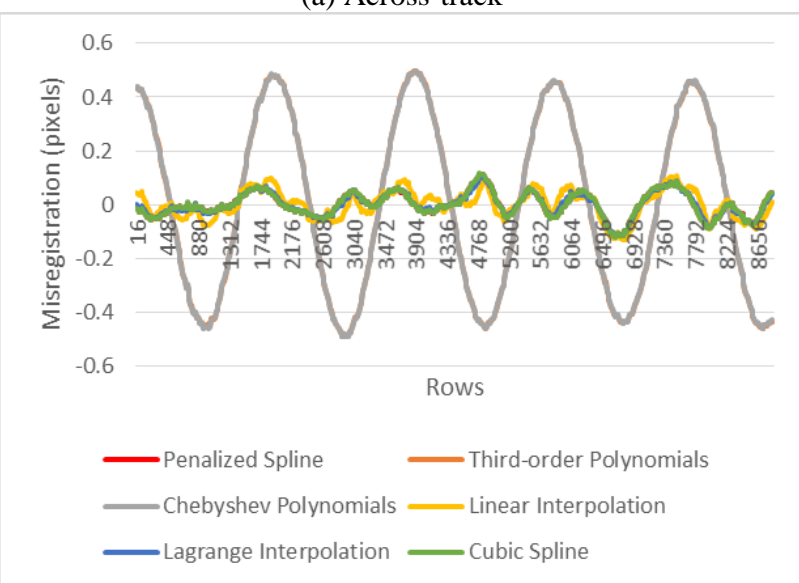

(b) Along-track

Figure 8 Band-to-band misregistration of the Dengfeng dataset caused by the attitude errors.

In the Dengfeng dataset, approximation models suffer from oscillation and its misregistration is much larger than that of interpolation models, which could model the oscillation. The penalized spline performs the same with cubic spline. And Lagrange interpolation got the best result, slightly better than the cubic spline and the penalized spline model.
Table 2 RMSE of misregistration of band 1 to band 3 caused by attitude errors.

\begin{tabular}{ccccc}
\hline \multirow{2}{*}{ Attitude Model } & \multicolumn{2}{c}{ Dengfeng } & \multicolumn{2}{c}{ Faizabad } \\
\cline { 2 - 5 } & $\begin{array}{c}\text { Across- } \\
\text { track }\end{array}$ & $\begin{array}{c}\text { Along- } \\
\text { track }\end{array}$ & $\begin{array}{c}\text { Across- } \\
\text { track }\end{array}$ & $\begin{array}{c}\text { Along- } \\
\text { track }\end{array}$ \\
\hline $\begin{array}{c}\text { Penalized Spline } \\
\text { Third-order } \\
\text { Polynomials } \\
\text { Chebyshev }\end{array}$ & 0.051 & 0.045 & 0.016 & 0.011 \\
$\begin{array}{c}\text { Polynomials } \\
\text { Linear }\end{array}$ & 0.568 & 0.329 & 0.046 & 0.018 \\
$\begin{array}{c}\text { Interpolation } \\
\text { Lagrange } \\
\text { Interpolation }\end{array}$ & 0.076 & 0.0529 & 0.046 & 0.017 \\
Cubic Spline & 0.051 & 0.045 & 0.037 & 0.034 \\
\hline
\end{tabular}

\section{CONCLUSIONS}

Building the trajectory model with discrete noisy data is a very hard task during the photogrammetric processing, because the real status of cameras in space are not clear. In this manuscript, we proposed the penalized spline model, a robust trajectory model, for ZY3 satellite, which could model the oscillation and overcome noises. The balance between the fitting accuracy and noise smoothing is controlled by a penalty parameter $\lambda$, which is estimated via the generalized cross-validation. The penalized spline model is compared with five other trajectory models, including third-order polynomials, Chebyshev polynomials, linear interpolation, Lagrange interpolation and cubic spline model.

Validation results show the penalized spline performs as well as Lagrange interpolation, cubic spline and Chebyshev approximation when sophisticated ephemeris is provided, and it outperforms other interpolation methods when on-board data are available. Meanwhile, its accuracy would not decrease with the increasing orbit length. In the case of standard scene, the third-order polynomials have the best result.

The band-to-band misregistration of ZiYuan-3 multispectral images is used to evaluate attitude models. The Dengfeng dataset and Faizabad dataset are used in the experiment. Results show that third-order polynomials and Chebyshev approximation could not model the oscillation of attitude. Large attitude errors would be introduced by the oscillating attitude. Lagrange interpolation shows a better performance than the linear interpolation and the cubic spline model. However, all the three interpolations above suffer from attitude noises. By contrast, the proposed penalized spline model could model the oscillation while be robust against noises.

\section{ACKNOWLEDGEMENTS}

This work was supported by the China Postdoctoral Science Foundation funded project 2015M572268 and the Fundamental Research Funds for Chinese Academy of Surveying \& Mapping.

\section{REFERENCES}

Breit, H., Fritz, T., Balss, U., Lachaise, M., Niedermeier, A., Vonavka, M., 2010. TerraSAR-X SAR Processing and Products. Geoscience and Remote Sensing, IEEE Transactions on 48, pp. 727-740.

de Boor, C., 2001. A Practical Guide to Splines Revised Edition. Springer. 
Dierckx, P., 1995. Curve and Surface Fitting with Splines. Clarendon Press.

Ebner, H., Kornus, W., Ohlhof, T., 1993. A simulation study on point determination for the MOMS-02/D2 space project using an extended functional model. International Archives of Photogrammetry and Remote Sensing 29, pp. 458-458.

Eilers, P.H., Marx, B.D., 1996. Flexible smoothing with Bsplines and penalties. Statistical science, pp. 89-102.

Golub, G.H., Heath, M., Wahba, G., 1979. Generalized CrossValidation as a Method for Choosing a Good Ridge Parameter. Technometrics 21, pp. 215-223.

Gugan, D.J., 1987. PRACTICAL ASPECTS OF TOPOGRAPHIC MAPPING FROM SPOT IMAGERY. The Photogrammetric Record 12, pp. 349-355.

Jeong, I.S., Bethel, J., 2010. A Study of Trajectory Models for Satellite Image Triangulation. Photogramm Eng Rem S 76, pp. 265-276.

Konecny, G., Lohmann, P., Engel, H., Kruck, E., 1987. Evaluation of SPOT imagery on analytical photogrammetric instruments. Photogramm Eng Rem S 53, pp. 1223-1230.

Li, R.X., Hwangbo, J., Chen, Y.H., Di, K.C., 2011. Rigorous Photogrammetric Processing of HiRISE Stereo Imagery for Mars Topographic Mapping. Ieee T Geosci Remote 49, pp. 2558-2572.

Luthcke, S.B., Zelensky, N.P., Rowlands, D.D., Lemoine, F.G., Williams, T.A., 2003. The 1-Centimeter Orbit: Jason-1 Precision Orbit Determination Using GPS, SLR, DORIS, and Altimeter Data Special Issue: Jason-1 Calibration/Validation. Marine Geodesy 26, pp. 399-421.

Pan, H., Zou, Z., Zhang, G., Zhu, X., Tang, X., 2016. A Penalized Spline-Based Attitude Model for High-Resolution Satellite Imagery. Ieee T Geosci Remote 54, pp. 1849-1859.

Radhadevi, P.V., Ramachandran, R., Murali Mohan, A.S.R.K.V., 1998. Restitution of IRS-1C PAN data using an orbit attitude model and minimum control. ISPRS Journal of Photogrammetry and Remote Sensing 53, pp. 262-271.

Schwind, P., Müller, R., Palubinskas, G., Storch, T., 2012. An in-depth simulation of EnMAP acquisition geometry. ISPRS Journal of Photogrammetry and Remote Sensing 70, pp. 99-106. Schwind, P., Schneider, M., Palubinskas, G., Storch, T., Muller, R., Richter, R., 2009. Processors for ALOS Optical Data: Deconvolution, DEM Generation, Orthorectification, and Atmospheric Correction. Geoscience and Remote Sensing, IEEE Transactions on 47, pp. 4074-4082.

SPOTImage, 2002. SPOT Satellite Geometry Handbook, S-NT73_12-SI, Edition 1, Revision 0.

Tanygin, S., 2003. Attitude Interpolation, AAS/AIAA Spaceflight Mechanics Meeting, Ponce, Puerto Rico.

Teshima, Y., Iwasaki, A., 2008. Correction of attitude fluctuation of terra spacecraft using ASTER/SWIR imagery with parallax observation. Ieee T Geosci Remote 46, pp. 222227.

Tong, X., Xu, Y., Ye, Z., Liu, S., Tang, X., Li, L., Xie, H., xie, j., 2015. Attitude Oscillation Detection of the ZY-3 Satellite by Using Multispectral Parallax Images. Geoscience and Remote Sensing, IEEE Transactions on 53, pp. 3522-3534.

Toutin, T., 2004. Review article: Geometric processing of remote sensing images: models, algorithms and methods. International Journal of Remote Sensing 25, pp. 1893-1924.

Weser, T., Rottensteiner, F., Willneff, J., Poon, J., Fraser, C.S., 2008. Development and testing of a generic sensor model for pushbroom satellite imagery. The Photogrammetric Record 23, pp. 255-274. 